Supporting Information

\title{
Interconnecting Bone Nanoparticles by Ovalbumin Molecules to Build a Three-Dimensional Low-density and Tough Material
}

Peter Samora Owuor ${ }^{1}$, Thierry Tsafack ${ }^{1}$, Hye Yoon Hwang ${ }^{1}$, Mohamed Sajadi ${ }^{1}$, Seohui Jung

${ }^{1}$,Tong $\mathrm{Li}^{2}$, Sandhya Susarla ${ }^{1}$, Bingqing Wei ${ }^{2}$, Robert Vajtai ${ }^{1}$,Jun Lou ${ }^{1}$, Sanjit Bhowmick ${ }^{3}$, Chandra Sekhar Tiwary* ${ }^{* 1,4}$,Pulickel M. Ajayan* ${ }^{1}$

${ }^{1}$ Department of Materials Science and NanoEngineering, Rice University, Houston, Texas 77005, USA

${ }^{2}$ University of Delaware, Delaware, 19716, USA

${ }^{3}$ Bruker Nano Surfaces, Minneapolis, MN, USA 55344.

${ }^{4}$ Metallurgical and materials Engineering, Indian Institute of Technology, Kharagpur, West Bengal, India 721302

Keywords: Hydroxyapatite, egg white, polymerization, 3D printing, primary amine Corresponding Authors: cst.iisc@gmail.com, ajayan@rice.edu 


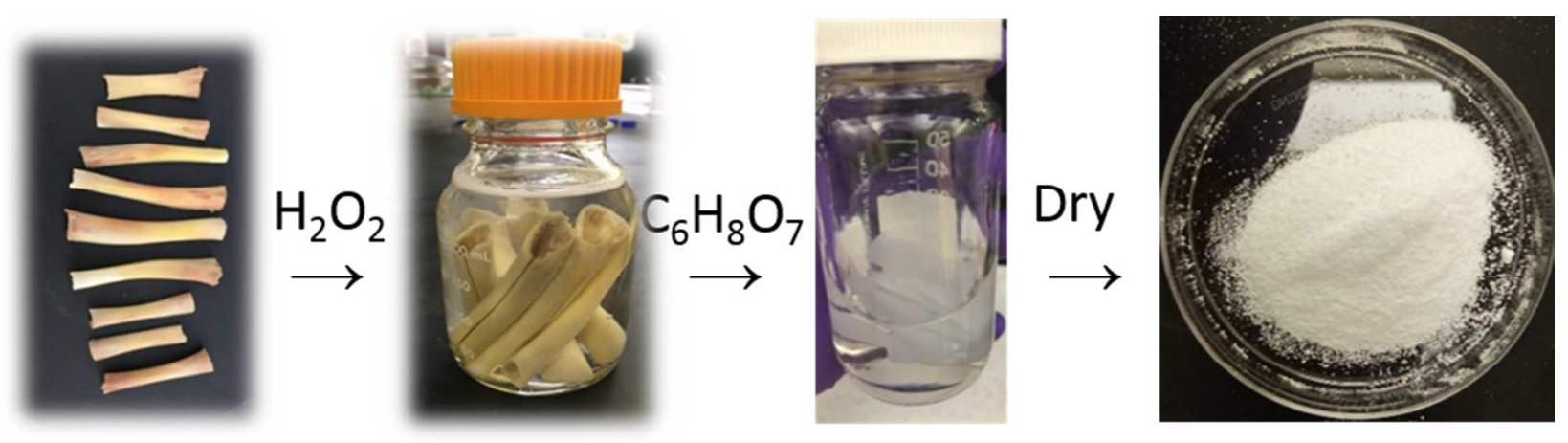

Fig. S-1 Procedure to remove inorganic bricks from bones.

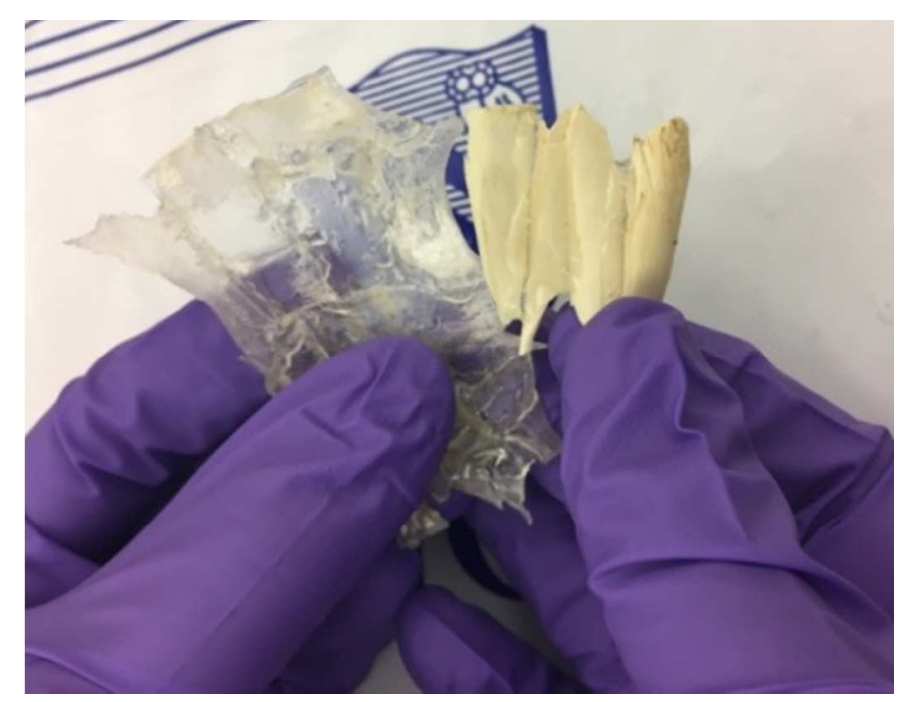

Fig. S-2 Intact transparent collagen structure after removal of nano-ceramics.

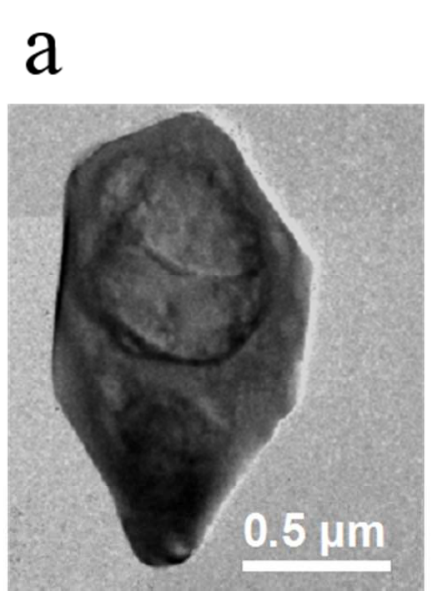

b

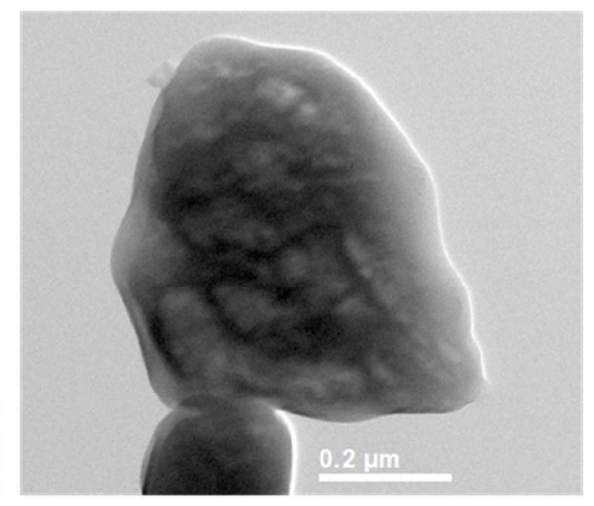

c

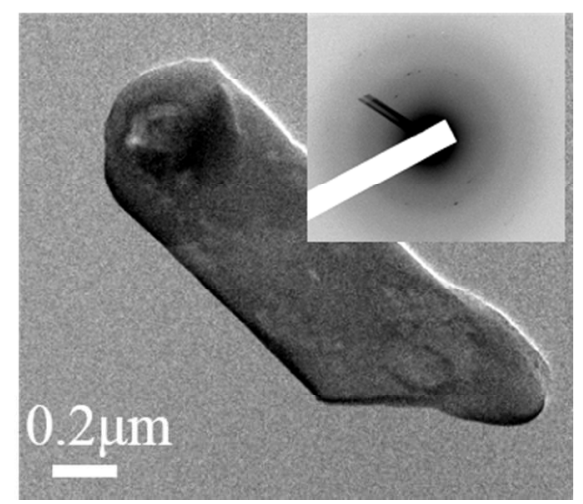

Fig. S-3 (a, b, c) Transmission electron microscope (TEM) images of hydroxyapatite crystals. 


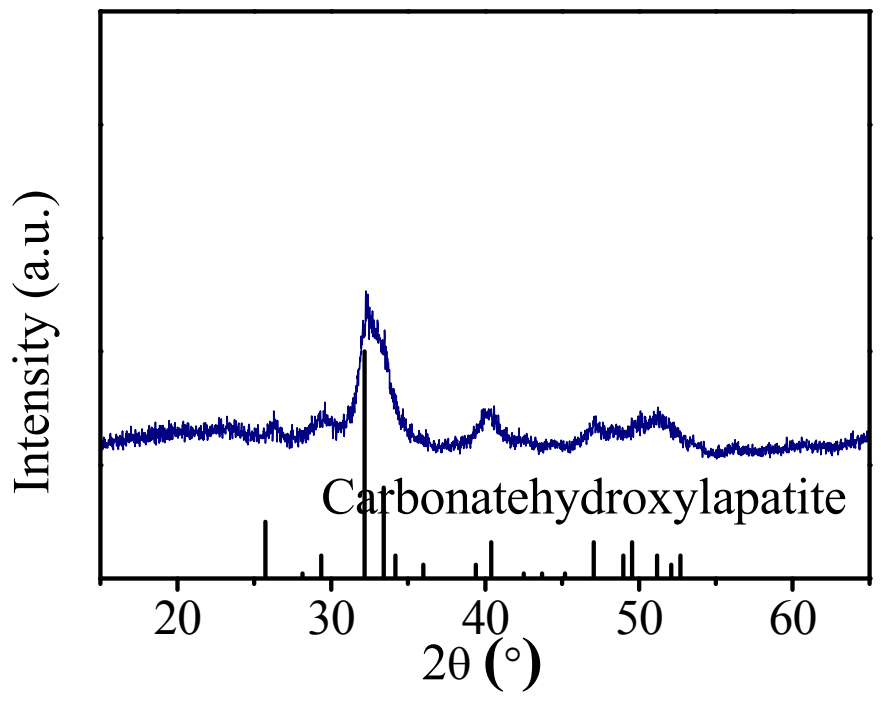

Fig. S-4: Polycrystalline nature of the nano-ceramics.

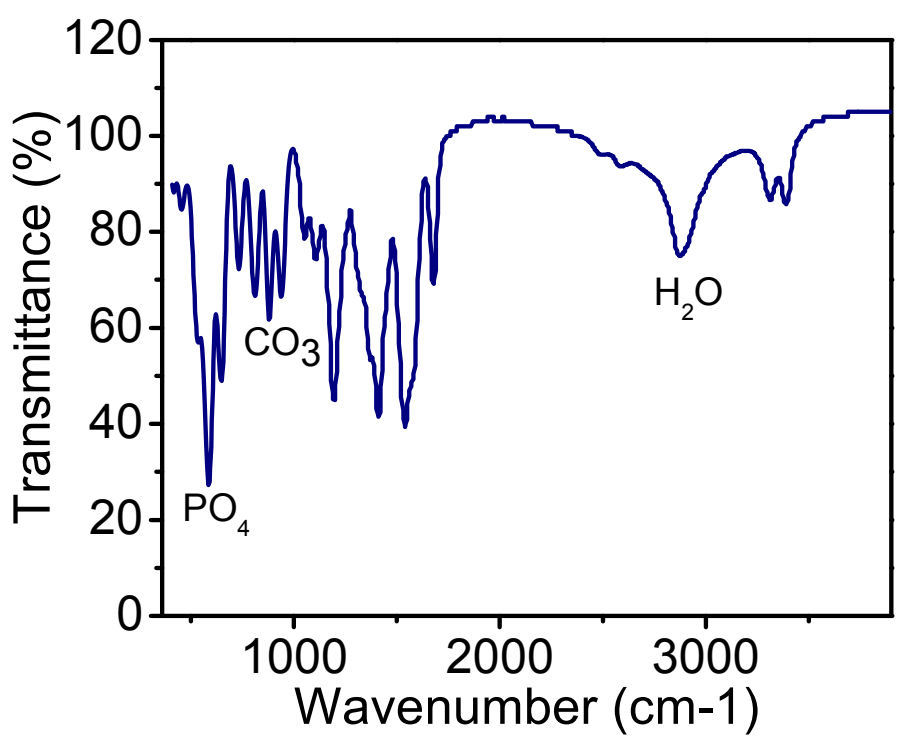

Fig. S-5. FTIR analysis of nano-ceramics. 

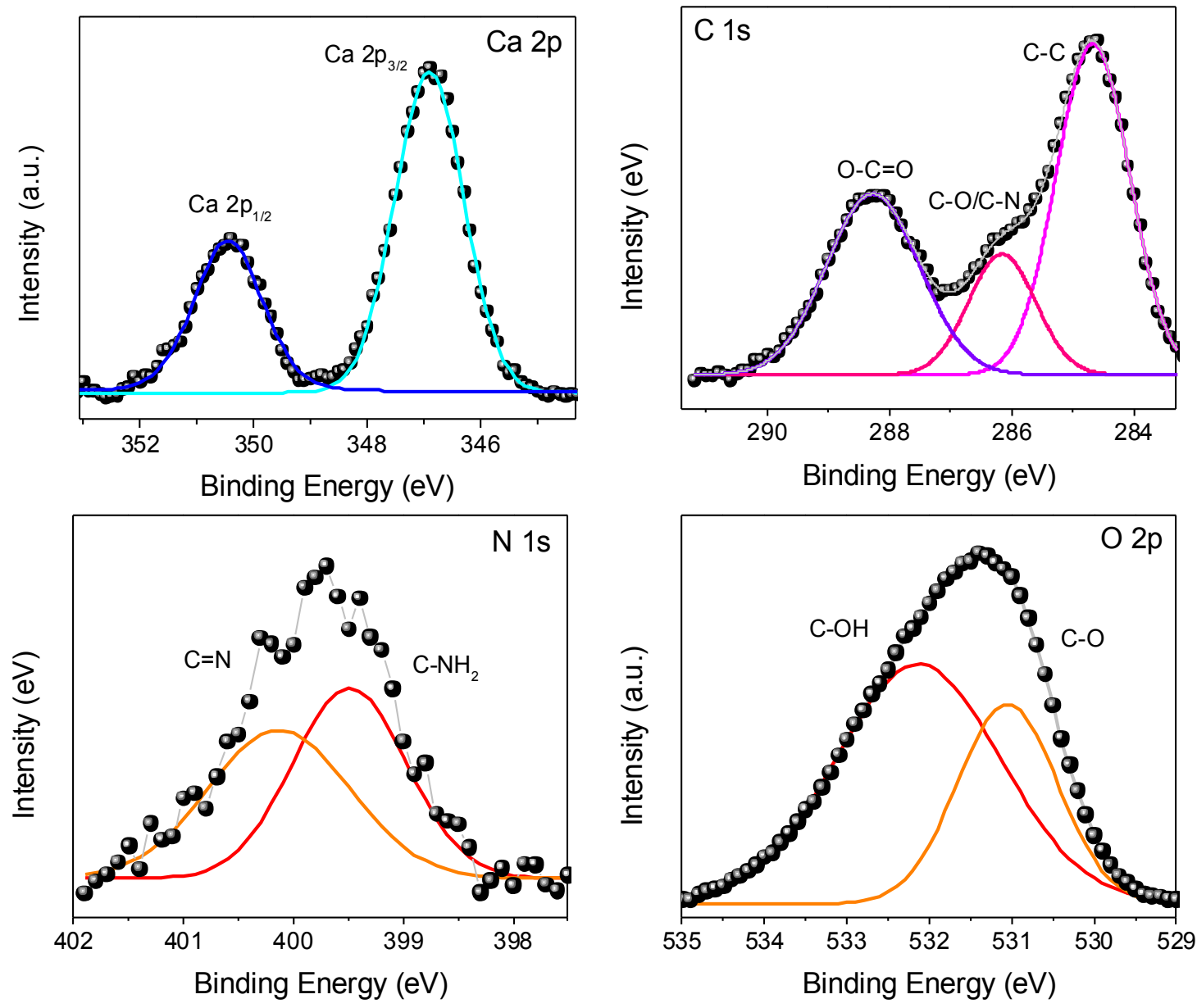

Fig. S-6. XPS fitting of hydroxyapatite.

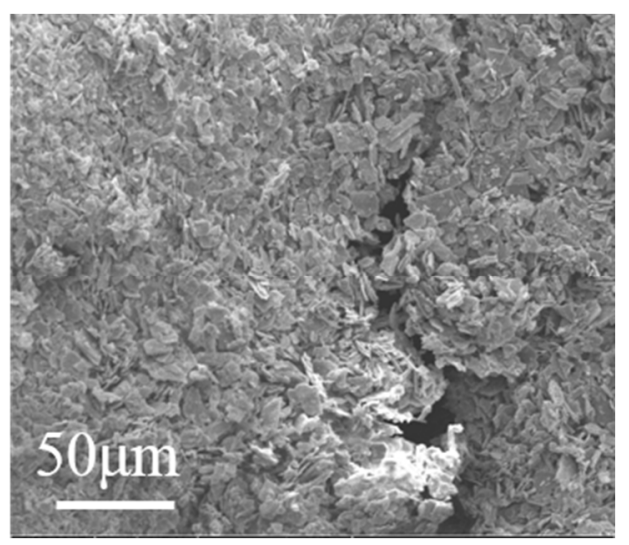

Fig. S-7 Cracks formation in pristine hydroxyapatite samples. 

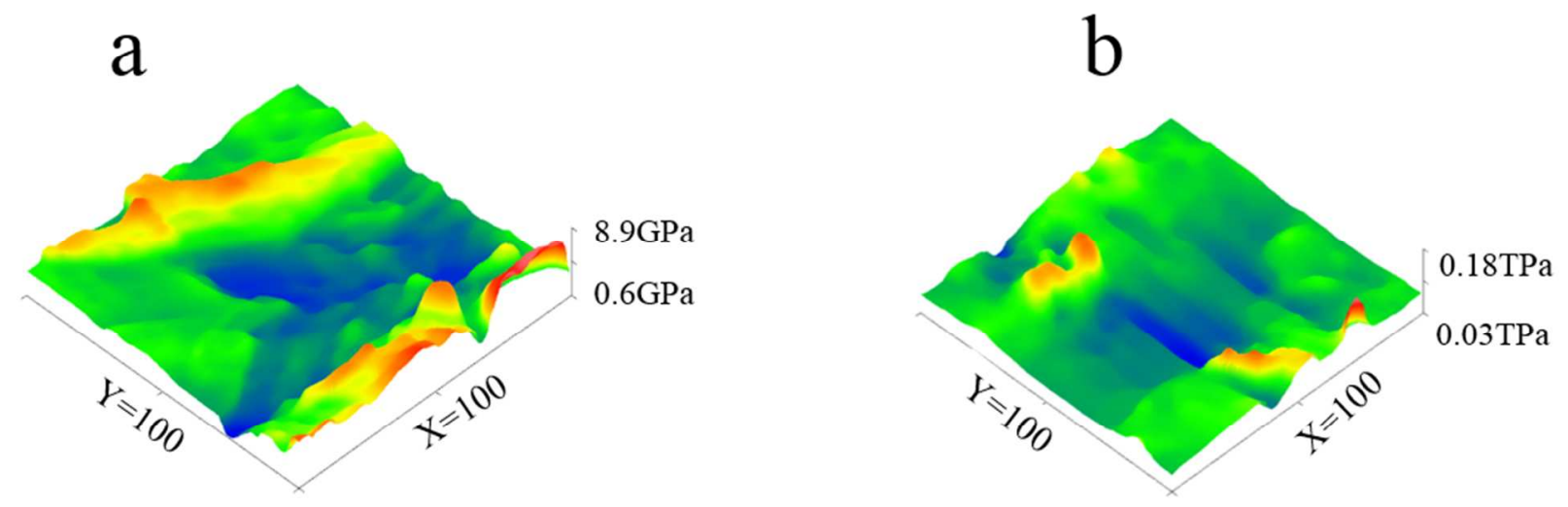

Fig. S-8 Three-dimensional modulus mapping (a) HA nano-ceramics powders with no egg white (b) HA/cross-linked egg white.

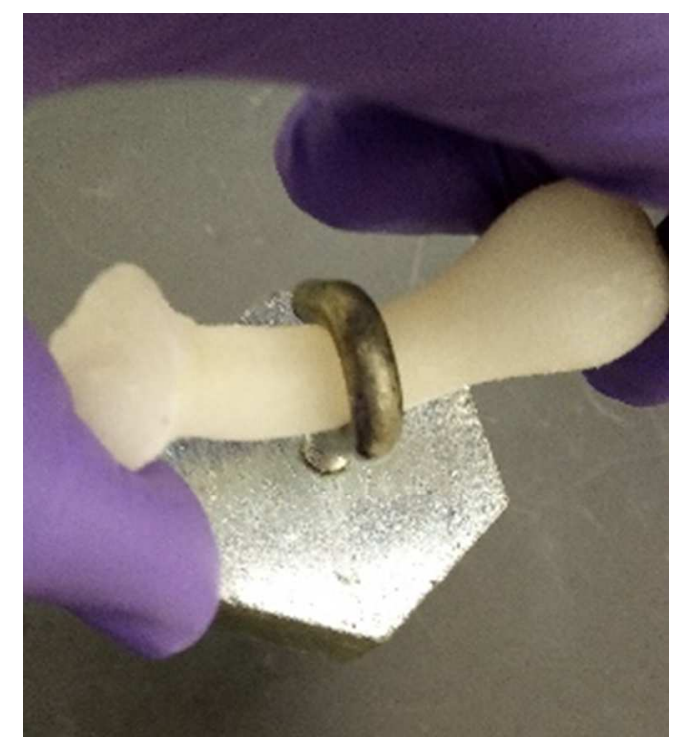

Fig. S-9 High strength of nano-ceramics/egg white composite carries a weight of $500 \mathrm{~g}$. 


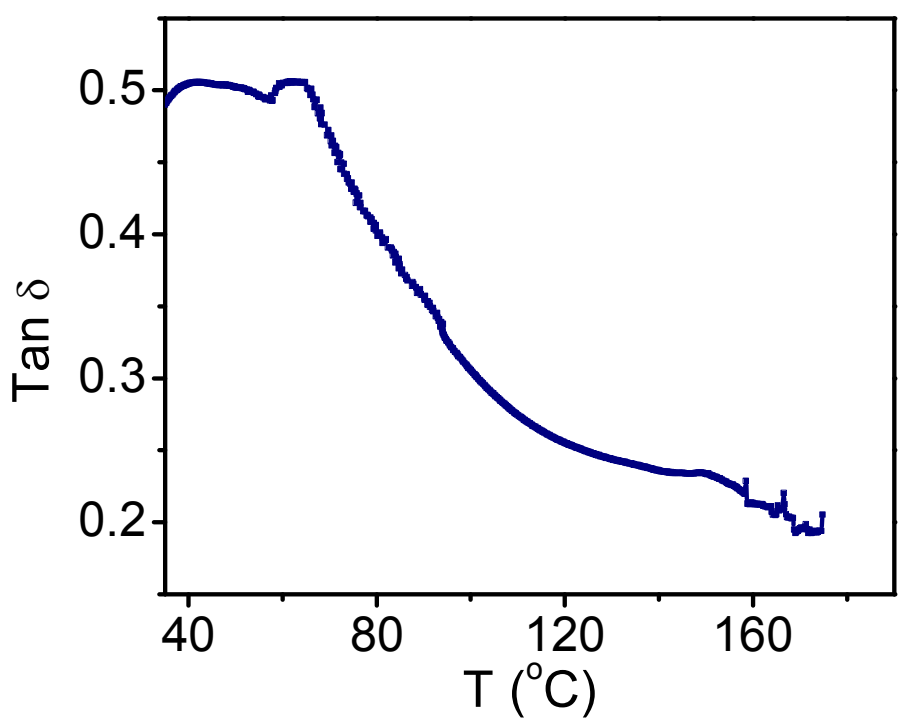

Fig. S-10 Tan delta values of nano-ceramics/egg white composite. 